\section{Medical meeting}

Medical Research Systems in Europe. Edited by E. P. Woodford. (A joint Wellcome Trust-Ciba Foundation Symposium held in London in March 1973. Ciba Foundation Symposium 21, New Series.) Pp. viii +335 . (Elsevier, NorthHolland: Amsterdam, London and New York, 1973.) Dfl. 43; \$17.20.

WITH all the turmoil in research circles following the British government's acceptance of the Rothschild "customer/ contractor" principle of research funding and organisation, this meeting was very timely. Twenty-two speakers from all the major countries in western and eastern Europe except for the Soviet Union circulated in advance papers describing the situation in their countries. This was a wise step, for though the papers are very useful sources of information, a repetition of the various types of support and organisation would have been tedious. The main purpose of the symposium was discussion, and this comprises some 80 pages of fascinating reading, indicating a real exchange of ideas.

Although the meeting was ostensibly about research, it also dealt fully with medical education and administration, and with the ways in which students could be channelled in to research careers. The most obvious differences between the different countries stemmed from their admission policies to medical schools. Most countries, both western and communist, now restrict their student entry to numbers near to those which the teachers think reasonable-the so-called "numerus clausus". Even so there were complaints of overcrowding and difficulties in communication. In Lisbon, where entry is unrestricted, a first-year entry of more than 2,000, with a $50 \%$ drop out, was found to be prejudicial to both research and teaching. Several speakers spoke of the danger to the community of the disappointed dropouts, though this seemed more serious to western than eastern participants. There was acceptance of high numbers of students by C. H. Nachev of Bulgaria, though the proportion receiving maintenance grants there is only $40 \%$ of that in Britain.

Nobody was entirely satisfied with the existing situation. Everybody deplored the lack of interest in research by many of the abler students, which seemed more serious than any shortage of funds. In all countries there was some division between work done in universities and separate institutes-where student numbers were high, institutes seemed to play the greatest part. Though the need to cooperate with scientists without clinical qualifications was realised, P. J. Hjort of Norway suggested, not entirely in fun,

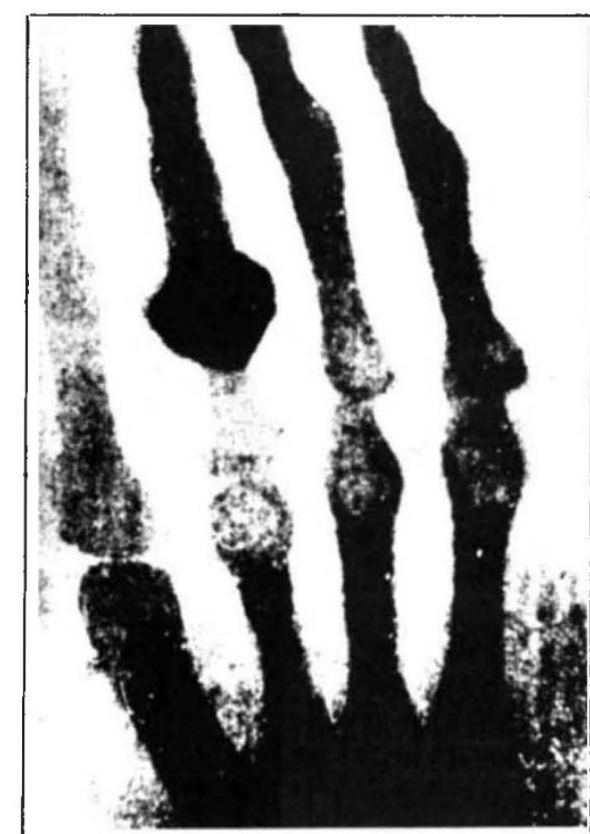

An early X-ray photograph of "the bones in the fingers of a living human hand. The third finger has a ring upon it." Described by W. C. Rontgen, writing on "a new kind of rays," in Nature in 1896. Reproduced in Patterns in Physics. By W. Bolton. Pp. vii +448 . (McGraw-Hill: Maidenhead, March 1974.) £3.95.

that, perhaps, "these invaders should be driven back, under the slogan "medicine for the doctors'". This sounded somewhat outdated to the ears of those from Britain, where the most eminent of the directors of our most prestigious medical research institutes have been, from a medical viewpoint, laymen. Clinicians and others regretted the difficulties of obtaining the cooperation of patients, though $\mathrm{H}$. Berndt said that in East Germany "patients are well educated and understand the value of clinical research, so they are cooperative".

The one thing missing from the symposium was any objective assessment of the success of any particular system of education and research. Experience elsewhere has indicated that there is little correlation between research productivity and available facilities. There was little which suggested that any political system was less-or more-likely to make research particularly productive. R. Saracci of Italy did indeed defend the principle of commissioned research, quoting Peter Medawar's point that painters and musicians in the past produced commissioned masterpieces, so "why shouldn't scientists react similarly?" This book was perhaps of greatest value in providing the background information for a survey of research efficiency, something which has still not been made, even by those who are responsible for the recent drastic changes in research organisation in Britain and elsewhere.

Kenneth Mellanby

\section{Introduction to superfluids}

Superfluidity and Superconductivity. By D. R. and J. Tilley. Pp. viii +262 . (Modern University Physics Series.) (Van Nostrand-Reinhold: New York and London, July 1974.) $£ 9.50$ cloth; $£ 4.95$ paper.

IT has long been recognised that the phenomenon of superfluidity in helium four has much in common with that of superconductivity in metals, each being marked by the macroscopic flow of material particles without dissipation of energy. A major defect of all previous introductory texts has been that although the similarities were in many cases commented upon, a genuinely integrated treatment was not attempted: it is this deficiency which the Drs Tilley have set out to rectify.

They start with brief descriptions of the observed properties of superfluid helium and of superconductors. The macroscopic wave function is then introduced on page 32 ; and it is around this unifying concept that the rest of the book is built, with chapters discussing the two-fluid model of HeII, electrodynamics and tunnelling in superconductors, vortex states, Josephson effects, Ginzburg-Landau theory, and the possibility of superfluidity in other systems.

The level assumes no more than an undergraduate knowledge of statistical mechanics, electricity and magnetism, and solid state physics, and the mathematical demands on the reader are remarkably modest. It is astonishing how much material the authors have managed to cram into the 248 pages of main text: I suspect that the large concentration of conceptual challenges, while highly stimulating to the beginning Ph.D. student, may prove rather strong meat for the majority of M.Sc. students.

A book of this scope is bound to attract minor quibbles from almost every reader whose particular research interest lies among the topics discussed. It does, however, seem rather a pity that, after a lucid exposition of Landau's famous explanation of superfluidity in terms of the elementary excitation spectrum, the authors fail to mention the one and only physical situation where this approach leads to an accurate numerical value of the critical velocity, namely, the passage of negative ions through pressurised HeII. On the other hand, I very much like the chapter on vortex states, a topic which lends itself particularly well to a unified treatment; and the section which presents a point by point comparison of vortices in superconductors with those in superfluid helium, emphasising the differences as well as the similarities, will be especially useful to novitiates. 
A measure of the continuing vigour of research in this field is given by the extent to which the book has been overtaken by important new discoveries during its writing. The most significant of these came with the observation in 1972 of two new and apparently superfluid phases of liquid helium three below $3 \mathrm{mK}$ thus, at a stroke, doubling the number of superfluids known in nature. The authors are to be commended for having managed to slip a page on this topic into the last chapter but, even so, the pace of advance has been such that already the passage strikes one as being principally of historical interest.

Vigorously and concisely written, the volume is lavishly adorned with diagrams and graphs of relevant experimental results, many of which will look comfortably familiar to workers in the field, but a significant number of which have not previously appeared within the pages of any textbook. There is a selection of problems at the end of each chapter except the last. In addition to references at the conclusion of each chapter, there is a select bibliography at the end of the book listing most of the standard texts and laced with descriptive comments, a number of which are amusingly barbed.

Thus in spite of one or two deficiencies, there is so much to be said on the positive side that $I$, for one, will strongly be recommending my graduate students and, indeed, some of my

\section{P. V. E. McClintock} colleagues to read this book.

\section{Analysis of rocks}

Laboratory Handbook of Petrographic Techniques. By Charles S. Hutchinson. Pp. xxvii +527 . (Wiley: New York and London, 1974.) $£ 10.60$.

THE title implies a rather unexciting prospect for the reader. Petrography is a vital part of petrology, and few scientists concerned with the genesis of rock complexes can afford to neglect the textures and mineral associations within a rock sample. Even so, it is the petrological analogue of anatomy in medicine, and has recently been in danger of neglect during the race by earth scientists to embrace geophysics and geochemistry as providing the main information for understanding the evolution of the Earth. Petrographic analysis is not essential for all geological studies, but there are few in which it does not demand an important role.

Some petrographic techniques have remained essentially $\mathrm{u} \mathrm{n} \mathrm{c}$ h a $\mathrm{nged}$ throughout this century, such as polarised-light microscopy, mineral separation, and determination of specific gravity and refractive index. There have, however, been advances in method and this book presents an upto-date survey of the whole field. The real advance made here, compared with earlier books on the subject, is the inclusion of sections on X-ray diff raction, differential thermal analysis and thermoluminescence measurements, and the expansion of the con-

\section{Please take note}

Effective Technical Writing and Speaking. By Barry T. Turner. Pp. xi+206. (Business Books: London, 1974.) £4.75.

THE Professor of Industrial Management at the University of Newcastle phrases, which is unfortunate because scientists in industry; there are chapters professorial licence perhaps.

on how to write specifications, sales There are a few misprints in the literature, contracts, patents and hand- book, some of which are amusing: books. But the sections on writing ("The medium is the massage"; Macarticles and reports, on organising Luhan). More serious is an error in a meetings, on giving talks and how best table on SI units, in which kilo, mega to illustrate them, will interest and giga imply factors of $10^{6}, 10^{9}$, and academics as well.

Professor Turner's advice on all ence books do not include a dictionary these topics is sensible and helpful. of quotations, which is perhaps why Some of it might even seem obvious, George Eliot is misquoted. And in a were it not for the incomprehensible book devoted to better writing the use handbooks, the turgid and jargon-filled of "disinterested" to mean "uninterarticles, and the ill-prepared lectures ested" is regrettable.

with illegible slides, that we are all un- In general, however, Professor happily familiar with.

Turner's book makes useful reading We are urged to avoid technical for anyone speaking or writing on jargon in our writing; to use short technical subjects. It would be a profitsentences and short Anglo-Saxon able addition to any library. words; and to approximate to con-

JOHN WALKER cept of petrography by including geochemical analysis by X-ray fluorescence, atomic absorption, and other techniques for components that cannot be analysed by physical methods. An excellent chapter on "Display of Data" deals rigorously with norm calculations, variation diagrams for rocks and minerals, and the statistical analysis of data.

The book is designed as a laboratory manual, and step-by-step instructions are given for all the techniques, ranging from photomicrography to the determination of feldspar structural state, matrix corrections in XRF spectrometry, or the calculation of mineral formulae. The subjects are well illustrated, and the book makes interesting reading. The author has, in fact, been remarkably sucessful in bringing new life to an old subject, probably because he conveys the impression that each technique has been subjected to his critical usage, and because he makes lively quantitative recommendations that are difficult to fault.

There is some overlap with Physical Methods in Determinative Mineralogy (edit. by J. Zussman) and Methods in Geochemistry (edit. by A. A. Smales and L. R. Wager), although surprisingly he does not refer to the latter. Nevertheless, the emphasis of this book is different and there is no real equivalent for a research student or advanced-level undergraduate specialising in petrology. It will save supervisors and tutors many hours of routine instruction, and may extend the life expectancy of much laboratory equipment.

My major criticism of this otherwise excellent book is the inclusion of 68 pages on X-ray fluorescence spectrometry which, like electron probe analysis, is dealt with in specialist texts. The author omitted electron probe techniques partly for that reason, whereas I would view them as vital to petrographic analysis. In contrast, the section on reflected-light microscopy is absurdly brief (about 6 pages) despite its vital role in petrography and the difficulties encountered by students.

Other notable omissions, in the context of the author's aims, are singlecrystal X-ray diffraction (for example anorthite structural state), petrofabric analysis, factor analysis, electron microscopy and Mössbauer spectroscopy. Some attention to problems of zoned crystals (refractive index section) and modal analysis (for example use of reflected light), and to mineral examples (eight pages for garnets versus one third of a page for clinopyroxeies, under $\mathrm{X}$-ray diffraction) are also needed, in any revised edition.

G. M. Brown 